\title{
Dynamic Provisioning of Resources in Hybrid Cloud using Aneka Platform
}

\author{
Prof. Sumalatha D. Bandari ${ }^{1}$, Anuradha A. Chavan ${ }^{2}$ \\ Professor, Department of CSE, AGTI'S Dr. Daulatrao Aher College of Engineering, Karad, India ${ }^{1}$ \\ Student, Dept of Information Technology, AGTI'S Dr. Daulatrao Aher College of Engineering, Karad, India ${ }^{2}$
}

\begin{abstract}
Many vendors are offering subscription-based computing services through the Infrastructure as a Service (IaaS) model. Users can purchase resources from different vendors and get the best from each of them to run their applications. But is very complex task to deploy applications in multi-cloud environments. Therefore, application platforms are necessary to help developers succeed. Developer use Aneka to deploy their applications in multi-cloud environments because Aneka provides platform as service. It can be used to provide resources from different cloud providers and can be configured to request resources dynamically according to the needs of specific applications. This paper introduces extensions built into Aneka to support the deployment of applications in multi-cloud environments. The first extension shows the flexibility of the Aneka architecture to add cloud providers. We also discussed the inclusion of public IPs to communicate resources located on different networks. We are reducing the total execution time of an application composed of independent tasks when deployed in the multi-cloud environment created by Aneka using Azure and EC2-supplied resources.
\end{abstract}

Keywords: IaaS, PaaS, Aneka, Map Reduce.

\section{INTRODUCTION}

In infrastructure-as-a-service (IaaS) Model, there are more than 50 vendors that offer computing Resources in the form of subscription for payment for use model. This poses a challenge in the selection of Cloud, but at the same time opens up an opportunity for applications can provide resources from multiple cloud providers. Running applications in cloud and multi-cloud environments brings significant benefits. However, they must be developed in order to exploit the main features of the cloud. Therefore, software applications must have the ability to acquire resources automatically without human intervention. According to their current needs, they should add and release resources dynamically by measuring and monitoring the use of these resources. Aneka is a platform as a service (PaaS) that helps Programmers to create applications that take advantage of clouds. It also helps developers build applications using different distributed programming models. It provides support for scheduling and deploying tasks on cloud resources and provides services to dynamically acquire and free these multi-cloud resources. Aneka components can be easily deployed to a virtual machine provided and support the monitoring of the resources in which applications are deployed. There are various public resource providers which work on the pay-per-use basis. Amazon EC2, GoGrid and Xen are the service providers that provide dynamic resources publicly in the form of pay-per-use.

Aneka allows exclusive kind of packages to be accomplished at the same grid infrastructure. A good way to guide such flexibility is to by provide the execution models. Currently Aneka supports three extraordinary execution models:

1. Task Execution model

Task programming affords developers with the ability of expressing packages as a set of impartial obligations. Every task can perform a special operation, or the equal operation on different data, and may be accomplished in any order by means of the runtime environment. That is a scenario in which many clinical applications suit in and a totally popular model for grid computing. This software model permits to speedy prototype disbursed applications with minimal implementation and management efforts and is specifically desirable for compute extensive programs where the computation can be prepared in numerous devices of execution. Also, challenge programming allows the parallelization of legacy applications at the cloud. Numerous software domains endorse challenges that may be solved through using mission programming, among them we will take into account clinical computing, monetary packages, media rendering and transcoding, and so on. This version is the most famous and well known in dispensed computing and may be used a starting point for implementing fashions with more complicated necessities along with workflows or parameter sweep.

\section{Thread Execution model}

This software version presents developers with the capability of strolling multithreaded applications on a allotted environment consisting of the Aneka cloud. The primary abstraction of this version is the concept of thread 
IARJSET

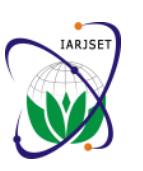

International Advanced Research Journal in Science, Engineering and Technology

National Conference on Innovative Applications and Research in Computer Science and Engineering (NCIARCSE-2017)

AGTI's Dr. Daulatrao Aher College Engineering, Vidyanagar Extension, Karad

Vol. 4, Special Issue 4, January 2017

which mimics the semantic of the common place nearby thread however is accomplished remotely in a disbursed environment. This model gives important manage on the execution of the single components of an software however calls for more control if compared with venture programming, which is based totally on a "put up and forget" sample.

A thread aid nearly all the operations available for local threads, extra specifically an Aneka thread has been designed to mirror the interface of the System.Threading.Thread.NET class, in order that developers can without difficulty move current multithreaded applications to the Aneka platform with minimal modifications. Preferably, packages can be transparently ported to Aneka simply with the aid of substituting neighbourhood threads with Aneka threads and introducing minimal modifications to the code. This model covers all of the utility situations of the task programming and solves the additional challenges of providing a distributed runtime surroundings for nearby multithreaded applications.

\section{MapReduce Execution version}

This version is an implementation of the MapReduce model, as proposed by means of google, for .net and integrated with Aneka. MapReduce has been designed to system a large amount of records by way of the use of simple operations that extracts beneficial facts from a dataset (the map function) and aggregates this facts collectively (the reduce function). Builders offer the good judgment for these two operations and the dataset and MapReduce will do the rest and made effects reachable while the application is completed. This utility version has often been named as the paradigm for developing programs for the cloud because its natural help for big dispensed infrastructure and ease of scalability in presence of huge quantities of facts. MapReduce may be a winning solution for records mining and analytic programs, bulk media processing, and content indexing. Aneka presents a strong help for the version and integrates it with all of the other foundation offerings which includes accounting and reporting, hence making this solution a aggressive opportunity in the identical marketplace phase.

\section{EXISTING SYSTEM}

Grid computing is the gathering of computer resources from a couple of locations to reach a common goal. The grid may be concept of as a disbursed system with noninteractive workloads that involve a huge quantity of documents.

Use of grids in scientific software brought about high usage fees along with technical and bureaucratic issues. For this vision to be done, however, middleware assisting provisioning of assets from each local infrastructures and public clouds (called hybrid clouds) is required, so that applications can transparently migrate to public digital infrastructures.

\section{BACKGROUND}

Multi-cloud applications

A multi-cloud platform is a middleware that allows the interconnection of cloud environments by using an individual layer that addresses the problems of integrating and provisioning resources from different cloud providers

- Access to more competitive prices:

Whatever prices offered for different configuration by providers can be compared and selected by the users. You can then choose the best configuration for your needs and the best price in the market for a specific configuration.

- High availability and improved response time:

When the resources are distributed in different clouds, user having the applications improves the response time and the availability of the applications.

- Fault tolerance and reliability:

The applications replicate across different cloud providers and different geographic regions, if one provider fails, the resources of another cloud provider can continue to respond to users' requests, this reduces the risk of failures.

- Not dependent on a cloud provider:

Applications that can be run on different cloud providers should be easily migrated, so if the user does not want to continue working with a specific provider, he can choose and move to a transparent one.

- Simplify premise and cloud integration

Applications that cannot be fully migrated to cloud environments can use multi-cloud platforms to provide the environment and support for successful integration with local resources.

\section{SYSTEM ARCHITECTURE}

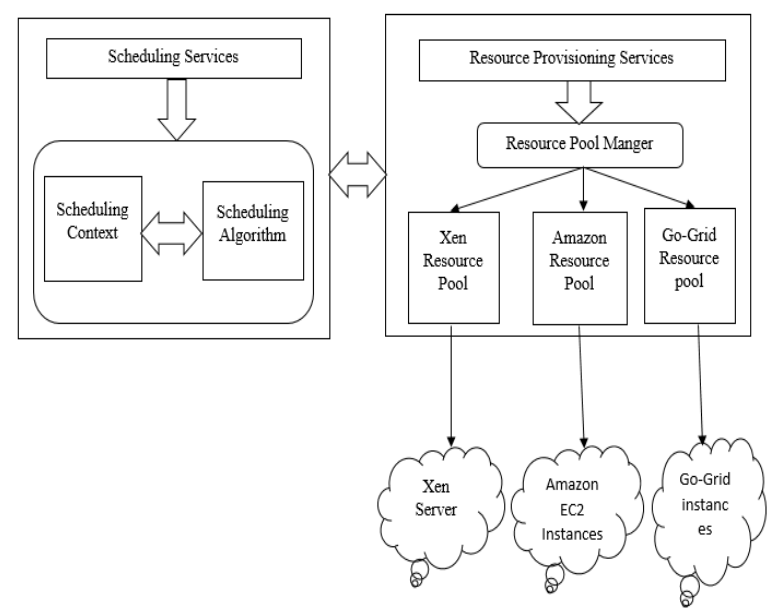

Fig.1 System Architecture

Aneka integrates dynamic provision capabilities as part of its provider version and dynamic provisioning is the end 
IARJSET

result of the collaboration of numerous services and various resource pools which provides the virtual additives that permit: resources.

1. Inquiring for digital device times from a selection of Amazon, Go-Grid and Xen provide Infrastructure-as-avirtual device managers or IaaS vendors. Service (IaaS). It delivers the set of services that which

2. Dynamically detecting the want of extra assets to keep forms a reliable, scalable, economically accessible cloud. the desired satisfactory of provider for utility execution.

3. Controlling the lease of dynamic assets to optimize their utilization.

These kinds of operations are transparently performed through the middleware without the consumer intervention and with a minimal configuration procedure throughout the Cloud setup.

- Scheduling Service:

Scheduling service manages the execution of applications for a specific programming model. According to the scheduling context scheduling service selects one of scheduling algorithm. The service provider is also selected which provides the efficient service for execution of application. Algorithm selected by the scheduling service reduces the overall execution time.

\section{- Scheduling Context:}

Schedule the application for execution, scheduling algorithm is used. It is in responsible for allocating a number of tasks to the available set of resources in a dynamic fashion. one scheduling algorithm for all the applications developed for a specific programming model and it is possible to select different algorithms while configuring the service. To schedule the available options, scheduling algorithm that supports dynamic provisioning is Deadline Priority Provisioning-Algorithm. This algorithm is leverage dynamic provisioning to cope with the application or system requirements.

- Resource Provisioning Services:

Resource provisioning infrastructure contains collection of resource pool which provides access to resource providers. The resource providers may be external or internal. All the resource providers are managed by resource pool manager. Resource provisioning service is provided by Aneka. Aneka container contains resource provisioning service and resource pool manager.

- Resource Pool Manager:

All the service providers are registered with resource pool manager and it allocates the required resources from those resource pools. It is an intermediator between the resource provisioning services and service provider. It request for resources from resource provider as per the requirement.

- Resource Pool:

Resource pool contains the virtual resources provided by same resource provider. It manages virtual resources it contains and release the resources which are no longer needed. As shown in Figure. 4.1 Xen resource pool, Amazon EC2 resource pool, Go-Grid resource pool are the

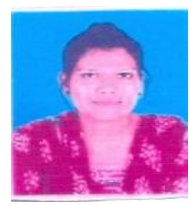

\section{CONCLUSION}

Cloud computing quick became the platform of preference in many realistic scenarios in a business context. Although, it's an adoption is restricted inside the context of computational technological know-how. Scientific applications requiring large amount of computing energy that may be introduced by using local resources inside a given time frame can utilize clouds that can supply this required capacity with minimal effort in phrases of the configuration of hardware structures. We are going to develop assist for the integration of multi-cloud. We presented the benefits of multi-cloud computing, access to competitive prices, high availability, fault redundancy and reliability, no dependency on a single cloud provider. To simplify the integration of resources from multiple cloud providers, we introduced the Aneka multi-cloud platform and its components that make it possible to run distributed applications in multi-cloud environments. In this context, the Aneka services described in detail about dynamic provisioning of resources. By using Deadline Priority Provisioning algorithm we execute high intensive applications in multi cloud environment within the given deadline. This reduces the total execution time require to complete the task.

\section{REFERENCES}

[1] Karthik Sukumar, Christian Vecchiola, Rajkumar Buyya "The Structure of the New IT Frontier: Aneka Platform for Elastic Cloud Computing Applications - Part III"

[2] Christian Vecchiola a, Rodrigo N. Calheiros a, Dileban Karunamoorthya, Rajkumar Buyyaa," Deadline-driven provisioning of resources for scientific applications in hybrid clouds with Aneka"

[3] Rajkumar Buyya and Diana Barreto, "Multi-Cloud Resource Provisioning with Aneka: A Unified and Integrated Utilisation of Microsoft Azure and Amazon EC2 Instances".

[4] Rajkumar Buyya "Cloud Computing Principles and Paradigams"

[5] C. Vecchiola, X. Chu, R. Buyya," Aneka: A Software Platform For .Net Based Cloud Computing, in: W.Gentzsch, L.Grandinetti, G.Joubert(Eds.)", 2009

[6] M.D.de Assuncao, A. di Costanzo, R.Buyya, "A Cost- Benefit Analysis Of Using Cloud Computing to Extend Capacity of Clusters, Cluster Computing" 2010

[7] Yi Wei, Karthik Sukumar, Christian Vecchiola, Dileban Karunamoorthy, Rajkumar Buyya "Aneka Cloud Application Platform and Its Integration with Windows Azure"

\section{BIOGRAPHIES}

Anuradha A. Chavan is perceiving BE from AGTI's Dr. Daulatrao Aher College of Engineering, Karad, India. Her area of interest Cloud Computing. 
IARJSET

Sumalatha D. Bandari received Diploma in Electronics \& Telecommunication Engineering from Government Polytechnic for Women, Nizamabad, Andhra Pradesh in 2001. B. Tech degree from Jawaharlal Nehru Technological University, Hyderabad in Computer Science \& Information Technology in 2005, the M. Tech degree from Jawaharlal Nehru Technological University, Hyderabad in 2011. From 2006 to 2010 she worked as an Assistant Professor in Department of Information Technology of Indur Institute of Engineering \& Technology, Siddipet, Andhra Pradesh. From 2011 to May 2012 she worked as an Assistant Professor in Department of Computer Science \& Engineering of Rajarambapu Institute of Technology, Sakharale, District Sangli, Maharashtra. Since June 2012 she is working as an Assistant Professor in Information Technology Department of Dr. Daulatrao Aher College of Engineering, Karad, District Satara, Maharashtra. Her area of interest includes Natural language Processing, Machine Learning \& Cloud Computing. 